\title{
Évaluation de la composition spécifique des glossines, vectrices de la Trypanosomose Humaine Africaine, dans la région de Ndendé au sud du Gabon
}

\author{
Christophe Roland ZINGA KOUMBA ${ }^{1}$, Franck MOUNIOKO ${ }^{2}$, Aubin Armel KOUMBA ${ }^{1 *}$, Julien Zahouli \\ BI ZAHOULI 3,4, Audrey Pamela MAROUNDOU 1, Geneviève ACAPOVI-YAO 4, Bertrand M'BATCHI 2, \\ Jacques François MAVOUNGOU 1,2 \\ 1 Institut de Recherche en Écologie Tropicale (IRET), BP : 13354, Libreville, Gabon. \\ 2 Université des Sciences et Techniques de Masuku, BP : 941, Franceville, Gabon. \\ ${ }^{3}$ Centre Suisse de Recherches Scientifiques en Côte d'Ivoire, 01 BP : 1303 Abidjan 01, Abidjan, Côte d'Ivoire. \\ ${ }^{4}$ Université Félix Houphouët-Boigny, UFR Biosciences 22, BP : 582, Abidjan 22, Côte d'Ivoire. \\ *Auteur correspondant: KOUMBA Aubin Armel \\ E-mail : aubinho25@yahoo.fr, Tel : 0024106661001
}

Original submitted in on $18^{\text {th }}$ January 2018. Published online at www.m.elewa.org on $31^{\text {st }}$ March 2018 https://dx.doi.org/10.4314/jab.v123i1.5

\section{RESUME}

Objectifs : Afin d'évaluer la composition spécifique des glossines dans les biotopes savanicoles et forestiers, une étude entomologique a été réalisée sur ces insectes dans la région de Ndendé (province de la Ngounié), ancien foyer de la Trypanosomose Humaine Africaine (THA) pour identifier les sites potentiels de transmission de cette parasitose.

Méthodologie et résultats : Un total de 14 pièges vavoua a été utilisé dont 7 pièges placés le long des cours d'eau présents dans la galerie forestière et 7 autres en savane proche des villages, du 23 octobre au 7 novembre 2015. Au total, 697 glossines ont été capturées (DAP=3,32 glossines/piège/jour). La plus forte abondance des glossines a été obtenue en forêt avec 678 spécimens. Cependant, ces glossines ont été faiblement capturées en savane (19 spécimens). Ces glossines se répartissent en quatre espèces dont Glossina fuscipes fuscipes, Glossina frezili, Glossina nashi et Glossina palpalis palpalis. G. fuscipes fuscipes (95\%) a été l'espèce la plus abondante alors que G. frezili (1\%), G. nashi (2\%) et G. palpalis palpalis (2\%) ont été très faiblement capturées.

Conclusion et application : Cette étude a permis d'identifier dans la région de Ndendé, quatre espèces de glossines qui vivent en sympatrie. Par ailleurs, la forte abondance de $G$. fuscipes fuscipes et la présence de $G$. palpalis palpalis, vecteurs de la THA dans les différents biotopes prospectés, avec une forte prédominance en forêt, suggèrent une existence probable d'un risque de transmission des trypanosomes dans la région de Ndendé. Par conséquent, le biotope forestier serait une zone prioritaire pour une lutte antivectorielle afin de limiter les risques de transmission de cette parasitose. Aussi, ces résultats invitent à la réalisation des travaux plus détaillés visant à identifier la dynamique spatio-temporelle des glossines et leur rôle dans la transmission éventuelle de la THA dans la région de Ndendé au sud du Gabon.

Mots-clés : Trypanosomose humaine africaine, glossine, composition spécifique, savane, forêt, Gabon. 


\section{ABSTRACT}

Objectives : In order to assess the species composition of tsetse flies among savannah and forested biotopes, an entomological survey was conducted on these insects in the Ndende region (province of Ngounié), ancient focus of Human African Trypanosomiasis (HAT) for identifying the potential sites of the HAT transmission. Methodology and results: A total of 14 Vavoua traps was used with 7 traps installed along the rivers present in the forest gallery and 7 others in the savannah near the villages. The study was done from $23^{\text {rd }}$ October to $7^{\text {th }}$ November 2015. In total, 697 tsetse flies were captured (ADT=3.32 tsetse flies/trap/day). The highest abundance of tsetse flies was found in the forest with 678 specimens, while the lowest abundance was observed in the savannah with 19 specimens. The identified tsetse flies are $G$. fuscipes fuscipes, $G$. nashi, $G$. frezili and G. palpalis palpalis. G. fuscipes fuscipes (95\%) was the most abundant species. Conversely, $G$. frezili (1\%), G. nashi (2\%) and G. palpalis palpalis (2\%) were less in number.

Conclusion and application: The results of this study showed the presence of four tsetse flies species, biological vectors of HAT, in the Ndendé region. The high abundance of $G$. fuscipes fuscipes and the presence of $G$. palpalis palpalis, vectors of HAT, in different savannah and forested biotopes, with strong predominance in forest, suggest a probable risk of transmission of trypanosomes in the Ndende region. Thus, the forested biotope would be the priority areas for implementing a vector control in order to limit the risks of HAT transmission. Therefore, these results require studies aiming at identifying spatial and temporal dynamics of tsetse flies and their role in the eventual transmission of HAT in the Ndendé region, southern of Gabon.

Keywords : Human African trypanosomiasis, tsetse fly, species composition, savannah, forest, Gabon.

\section{INTRODUCTION}

La Trypanosomose Humaine Africaine (THA) ou maladie du sommeil est une affection parasitaire transmise par les glossines ou mouches tsé-tsé et causée par la présence dans le sang, la lymphe et/ou le liquide céphalo-rachidien d'un protozoaire flagellé sanguicole exoerynthrocytaire appelé trypanosome (Aksoy, 2003 ; Mbida et al., 2009, Peka et al., 2014). Cette parasitose est endémique dans de nombreux pays d'Afrique centrale du fait de la présence de plusieurs espèces du genre Glossina qui en assurent la transmission à l'homme (Aksoy, 2003, Courtin et al., 2005 ; Mbida et al., 2009; Bosson-Vanga et al., 2012 ; Dibakou et al., 2015). Selon l'Organisation Mondiale de la Santé (OMS), la THA est à l'origine de $87 \%$ du nombre total de malades diagnostiqués dans toute l'Afrique (WHO, 2006). Actuellement, environ 60 millions de personnes sont exposées à cette parasitose et on estime à 9878 celles qui en sont atteintes (OMS, 2015). De plus, ces zoonoses figurent parmi les principales causes d'hospitalisation, de morbidité et de mortalité dans de nombreux pays (OMS, 2016). Par ailleurs, les maladies provoquées par les Trypanosomatidae appartiennent au groupe des maladies tropicales négligées (MTN) définies par I'OMS (Milleliri et al., 2009). Ces maladies sont fortement ré-émergentes et ont un impact considérable sur la santé publique et le développement économique des pays d'Afrique subsaharienne (Milleliri et al., 2009; Seck et al., 2010; Allou et al., 2015). Au Gabon, les connaissances portant sur les glossines, vecteurs biologiques de la THA, et la transmission de cette pathologie demeurent insuffisantes dans certaines localités, en particulier la région de Ndendé (Zinga et al., 2014 ; Doumba et al., 2016). En effet, de nombreux foyers historiques n'ont pas été prospectés depuis plus de 15 ans en raison de la faiblesse des moyens financiers du Programme National de Lutte contre la Trypanosomiase Humaine Africaine (PNLTHA) (Kohagne et al., 2011). Pourtant, les études conduites dans certaines provinces, dont celle de l'Ogooué-Maritime, ancien foyer historique de la THA, ont permis de mettre en évidence la présence des glossines infectées par les trypanosomes (Kohagne et al., 2011 ; Zinga et al., 2015). Aussi, des cas sporadiques de THA sont diagnostiqués de manière passive par les structures sanitaires de la ville de Port-Gentil dans la province de l'Ogooué-Maritime (Amblard, 1996 ; Milleliri et al., 2009). Dans la province de la Ngounié et, en particulier, dans la région de Ndendé, les données 
relatives aux glossines, vecteurs biologiques de la THA, sont presqu'inexistantes (Doumba et al., 2016). Pourtant, cette région est caractérisée par une succession de mosaïques forêt-savane, des galeries forestières et de milieux anthropisés, notamment de nombreux villages favorables au développement des mouches piqueuses dont les glossines (Doumba et

\section{MATERIEL ET METHODES}

Zone d'étude : La présente étude a été réalisée dans la région de Ndendé située dans la province de la Ngounié, au Sud du Gabon (Figure 1). La localité de Ndendé est al., 2016). Ainsi, une étude entomologique basée sur l'inventaire de ces glossines a été conduite dans les différents biotopes caractéristiques de la région de Ndendé. L'objectif de cette étude était de déterminer la composition spécifique (la diversité et l'abondance) des glossines dans les zones de savane et les forêts galeries.

située à $75 \mathrm{Km}$ de la ville de Mouila et à $549 \mathrm{Km}$ de la capitale Libreville.

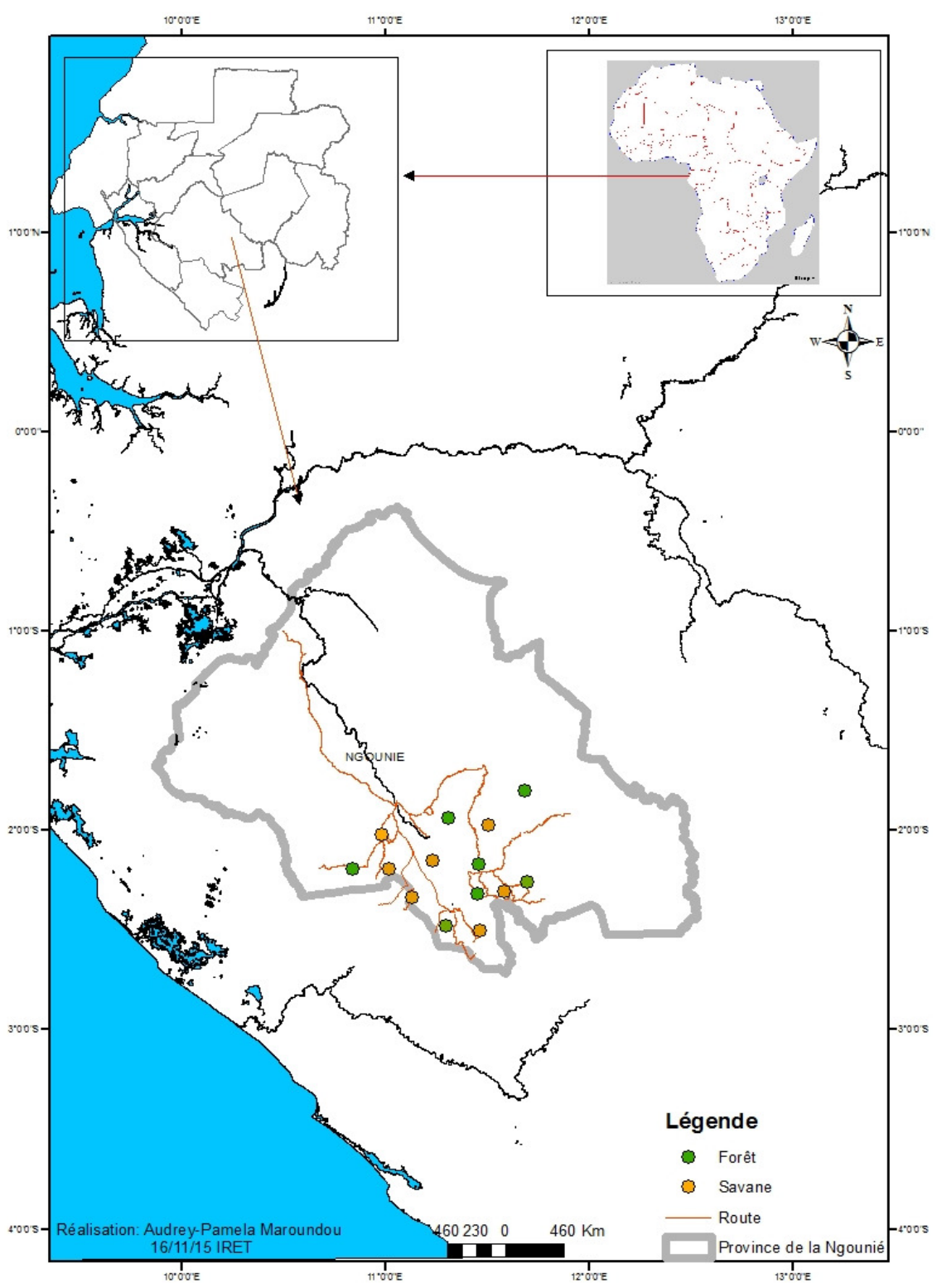

Figure 1 : Localisation de la zone d'étude et des sites de captures des glossines 
Cette région est soumise à un climat équatorial, humide et chaud (Collinet et Forget, 1976) caractérisé par une saison sèche de 3 mois (juin à août), et d'une saison des pluies de 8 à 10 mois (octobre à mai). La saison sèche est marquée par une quasi absence des pluies alors que la saison pluvieuse est caractérisée par des précipitations comprises entre 1800 et $2200 \mathrm{~mm}$ (Edou, 2012). La végétation de la région de Ndendé est composée essentiellement des savanes et des galeries forestières. Les savanes sont plus ou moins arbustives avec des plantes herbacées dont Pobeguinea arrecta, Hypparhenia rufa et Hypparhenia diplandra. Ces diverses espèces forment des peuplements denses alors que certaines plantes telles que les Andropogoneae constituent des peuplements peu denses. Par contre, les forêts galeries colonisent principalement les abords des cours d'eau. Elles abritent un grand nombre d'espèces végétales dont les plus importantes sont Uapaca guineensis, Sarcocephalus pobeguini, Aucoumea klaineana, Symphonia giobuligera. Ces espèces sont souvent adaptées aux conditions hydromorphes (Doumba et al., 2016). Cependant, dans les zones de transition forêtsavane, on rencontre des couronnes de végétation plus hautes et plus denses. Les fougères grand-aigle (Pteridium aquilinum) y sont particulièrement abondantes. La faune terrestre de cette localité est riche et représentée par plusieurs espèces animales telles que les singes à queue de soleil (Cercopithecus solatus), les gorilles (Gorilla gorila), les chimpanzés (Pan troglodytes), les buffles (Syncerus caffer nanus), les éléphants (Loxodonta africana), les céphalophes à pattes blanches (Cephalophus ogilbi crysalbum). De même, cette région héberge de nombreuses espèces d'oiseaux, de petits mammifères, de reptiles, d'amphibiens et d'insectes (Doumba et al., 2016). Le réseau hydrographique est dominé par la rivière Ngounié, qui est le cours d'eau le plus important servant d'axe d'écoulement et de niveau de base à toute la région. Les axes secondaires sont souvent interrompus par des dépressions plus profondes ne débordant qu'en fin de saison des pluies (Doumba et al., 2016).

Capture des glossines : Les glossines ont été capturées à l'aide des pièges Vavoua (Laveissière et Grébaut, 1990), du 23 octobre au 7 novembre 2017 (15 jours), au cours de la saison des pluies. Ces pièges de type Vavoua sont des pièges monoconiques mis au point par Laveissière et Grébaut en 1990, dans le foyer forestier de Vavoua en Côte d'Ivoire pour lutter contre les glossines. Ainsi, un total de 14 pièges a été utilisé au cours de la présente étude dont 7 pièges ont été placés le long des cours d'eau présents dans la galerie forestière et 7 autres en savane proche des villages. Les pièges étaient distants les uns des autres d'au moins $500 \mathrm{~m}$ et chacun constituait un point de capture. Par ailleurs, les pièges ont fonctionné en continu durant toute la période d'étude. Cependant, les relevés quotidiens des pièges se faisaient à 7 heures et à 18 heures. Aussitôt, les glossines capturées ont été ramenées au laboratoire pour leur tri et leur identification.

Identification morphologique des espèces de glossines capturées: Les différentes espèces de glossines collectées ont été identifiées à partir des caractères morphologiques (coloration des tarses et de l'abdomen) et de la structure des génitalia (organes génitaux externes) en utilisant les clés d'identification de Pollock (1982) et de Brunhes et al. (1998).

Analyse statistique des données: L'abondance des glossines a été définie par la densité apparente par piège et par jour (DAP) et calculée selon la formule suivante :

\section{$D A P=\frac{\text { Nombre de mouches capturées }}{\text { Nombre de pièges } x \text { nombre de jours de capture }}$}

La diversité spécifique des glossines dans les milieux étudiés a été déterminée par l'indice de diversité de Shannon (Shannon, 1948) à partir de la formule suivante $: H^{\prime}=-\Sigma(\mathrm{Ni} / \mathrm{N}) \times \log (\mathrm{Ni} / \mathrm{N})$ où $\mathrm{Ni}$ représente le nombre d'individus d'une espèce donnée et $\mathbf{N}$ le nombre total d'individus. L'indice de Simpson qui permet de calculer la probabilité que deux individus sélectionnés aléatoirement dans un milieu donné soient de la même espèce (Simpson, 1949), a été calculé suivant la formule :
$\mathrm{D}=\sum \mathrm{Ni}(\mathrm{Ni}-1) / \mathrm{N}(\mathrm{N}-1)$, avec $\mathbf{N i}:$ nombre d'individus de l'espèce donnée et $\mathbf{N}$ : nombre total d'individus.

Par ailleurs, le test $t$ a été effectué pour comparer la distribution des différentes espèces de glossines capturées en fonction des biotopes prospectés. Le logiciel utilisé pour faire les comparaisons était XLSTAT 7.5. Le test était significatif lorsque $P$ était inférieur à $5 \%(P<$ 0.05). 


\section{RESULTATS}

Abondance des glossines : Au total, 697 glossines ont été capturées dans la zone d'étude, soit une DAP de 3,32 glossines/piège/jour (G/P/J). Un total de 678 spécimens (97\%) a été identifié en forêt et 19 spécimens (3\%) en savane. Les DAP ont été plus élevées en forêt et plus faibles en savane, avec respectivement des valeurs de 6,46 et $0,18 \mathrm{G} / \mathrm{P} / \mathrm{J}$.

Composition spécifique des glossines: Les spécimens de glossines collectés ont été répartis en quatre (4) espèces et regroupés en deux sous-genres (Austenina et Nemorhina) (Tableau 1). Les espèces de glossines appartenant au sous-genre Austenina ont été représentées par Glossina frezili et Glossina nashi. Quant aux glossines du sous-genre Nemorhina, elles ont été composées de Glossina fuscipes fuscipes et Glossina palpalis palpalis.

Tableau 1 : Sous-genres et espèces de glossines répertoriés dans la région de Ndendé

\begin{tabular}{c|c}
\hline Austenina & Nemorhina \\
\hline Glossina frezili & Glossina fuscipes fuscipes \\
\hline Glossina nashi & Glossina palpalis palpalis \\
\hline
\end{tabular}

G. fuscipes fuscipes (95\%; $n=697$ ) a été l'espèce la plus abondante avec une DAP de 3, $15 \mathrm{G} / \mathrm{P} / \mathrm{J}$ (Figure 2). Les autres espèces, à savoir G. frezili (1\%), G. nashi $(2 \%)$ et
G. palpalis palpalis $(2 \%)$ ont été très faiblement représentées avec des DAP respectives de 0,04, 0,06 et $0,07 \mathrm{G} / \mathrm{P} / \mathrm{J}$.

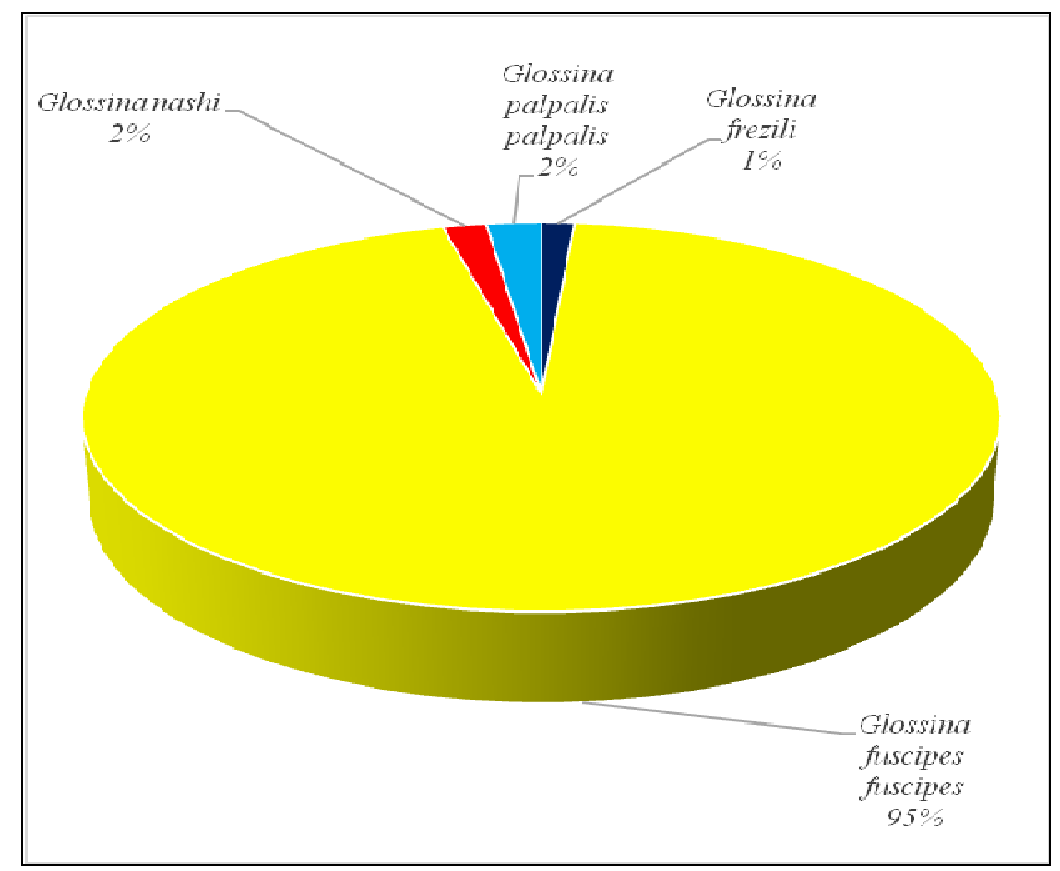

Figure 2 : Abondance des espèces de glossines capturées dans la région de Ndendé

Composition spécifique des glossines en fonction des biotopes prospectés : L'abondance des glossines capturées a été plus élevée en forêt $(97 \% ; n=678)$ qu'en savane $(3 \% ; n=19)$. En forêt, 4 espèces de glossines ont été capturées dont G. frezili, G. nashi, G. fuscipes fuscipes et $G$. palpalis palpalis. G. fuscipes fuscipes a été l'espèce la plus abondante en forêt avec une DAP de 6,15 G/P/J. Cependant, les autres espèces ont été très faiblement représentées avec des DAP inferieures à $1 \mathrm{G} / \mathrm{P} / \mathrm{J}$ en forêt (Figure 3). En savane, 4 espèces ont été également identifiées avec des abondances différentes. En effet, ces espèces ont été représentées par G. frezili, G. nashi, G. palpalis palpalis et $G$. fuscipes fuscipes avec des DAP respectives de $0,01,0,01,0,02$ et $0,14 \mathrm{G} / \mathrm{P} / \mathrm{J}$ (Figure 3 ). Tout comme en forêt, $G$. fuscipes fuscipes a été l'espèce la plus abondante en savane. Par contre, les autres espèces ont été très faiblement présentes dans ce biotope. 


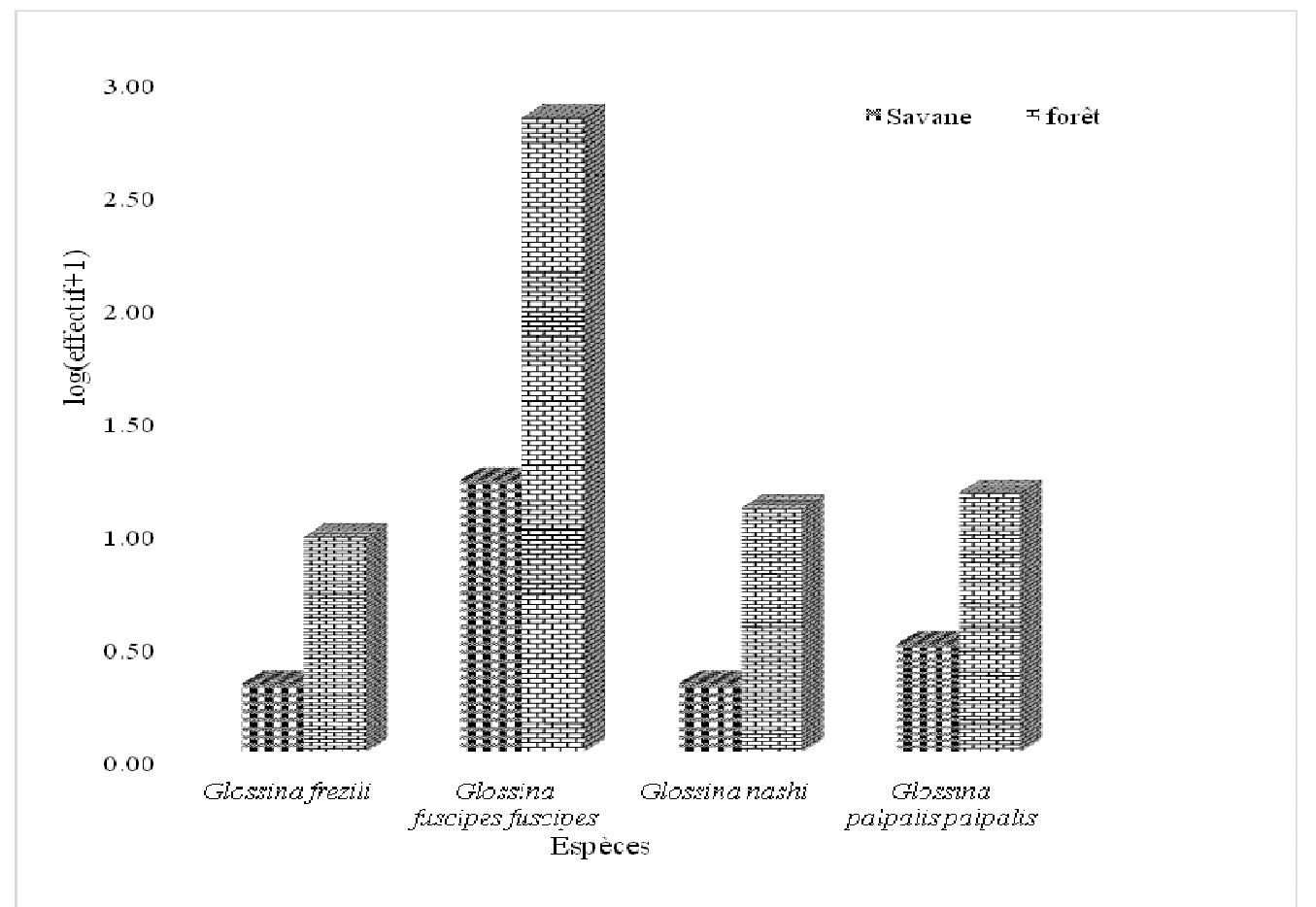

Figure 3 : Répartition des espèces de glossines en fonction des biotopes prospectés

La diversité des populations de glossines dans les différents biotopes a été évaluée par l'indice de Shannon. Les valeurs de cet indice ont été de 0,10 en forêt et 0,32 en savane. La dominance des espèces de glossines était substantiellement plus élevée en forêt $(D=0,91)$ comparativement à la savane $(D=0,61)$. Cependant, les

\section{DISCUSSION}

Les résultats obtenus dans cette étude constituent des données actualisées sur les vecteurs biologiques de la trypanosomose humaine africaine dans la région de Ndendé (Sud du Gabon). De plus, il n'existe aucune information sur la répartition biogéographique de ces insectes dans cette localité (Doumba et al., 2016). Ces résultats ont permis de mettre en relief la présence de quatre espèces de glossines (G. frezili, G. nashi, $G$. fuscipes fuscipes et $G$. palpalis palpalis) qui vivent en sympatrie dans les zones prospectées avec des DAP variables suivant l'espèce. Par ailleurs, cet inventaire d'espèces de glossines pourrait servir de base pour les études complémentaires visant à mettre en place la lutte anti-vectorielle adaptée à chaque type de biotope. Les pièges Vavoua qui ont été utilisés au cours de cette étude ont présenté l'avantage de capturer le groupe cible (les glossines). Toutefois, les faibles captures qui ont été enregistrées au cours de ce travail pourraient être liées à l'utilisation exclusive du piège Vavoua, mais aussi à la résultats du test $t$ ont révélé qu'il n'y a aucune différence significative $(p=0,340 ; d d l=6)$ dans la distribution des différentes espèces de glossines capturées entre les deux biotopes.

période de capture des glossines et au faible nombre de sites prospectés. De ce fait, les résultats de cette étude ne sont pas exhaustifs. En effet, bien que l'efficacité des pièges vavoua pour la capture des glossines soit largement admise (Laveissière et Grébaut, 1990 ; Gilles, 2005; Gilles et al., 2007; Mavoungou et al., 2012; Zinga et al., 2013a ; Zinga et al., 2013b ; Dibakou et al., 2015; Mounioko et al., 2015; Zinga et al., 2015 ), il n'en demeure pas moins que l'association de plusieurs types de piège (Nzi, grand Tetra, petit Tetra, Vavoua) et l'adjonction d'attractifs olfactifs comme l'octénol seul (Jaenson, 1991; Mihok et al., 1995) ou en association avec des phénols (Mihok et al., 1995) auraient probablement permis d'améliorer la qualité et la quantité des captures (Djiteye, 1992; Amsler et Fillerdier, 1994). Par ailleurs, la période de capture des mouches (octobre à novembre) correspond à la grande saison des pluies. Cette saison est caractérisée par de nombreux orages accompagnés de fortes précipitations qui sont néfastes à 
la dynamique des populations adultes de glossines. En plus, ces fortes pluies peuvent engendrer une dormance temporaire des larves lorsque leurs gîtes sont inondés (Raymond, 1980; Desquesnes et al., 2005). Ces résultats sont similaires à ceux obtenus par Mavoungou et al. (2012) qui ont montré l'impact des précipitations sur l'abondance des mouches hématophages dans la région de Makokou au nord-est du Gabon. L'abondance et la répartition des différentes espèces de glossines n'ont pas été uniformes et ont varié en fonction des biotopes prospectés. En effet, l'abondance des glossines a été plus importante en galerie forestière qu'en savane. Cette distribution peut être due à la stabilité du milieu forestier (omniprésence de l'humus, température plus ou moins constante, présence de rivière, etc.) et à l'abondance de la grande faune, hôte nourricier des insectes hématophages dans ce biotope (Broce et al., 2005), contrairement à la savane où l'on note une fréquentation erratique et temporaire de la faune vertébrée. Cette répartition semble également être influencée par la différenciation des paysages et la structuration des milieux prospectés pouvant engendrer des microhabitats propices au développement des glossines (Dibakou et al., 2015 ; Mounioko et al., 2015). Par exemple, la galerie forestière est caractérisée par des grands arbres dont les cimes forment une canopée qui obscurcit le sous-bois et atténue considérablement la température (Mavoungou, 2007 ; Sima et al., 2018). En revanche, la savane présente une végétation plus basse et moins fournie et, les températures y sont relativement plus élevées en relation avec un temps d'éclairement plus important. La conjugaison de plusieurs facteurs tels qu'une température comprise entre 15 et $25^{\circ} \mathrm{C}$, une végétation dense et la présence d'hôtes nourriciers en abondance serait à l'origine de la forte densité de glossines dans une zone donnée (Laveissière et Hervouët, 1991; Gouteux et Kiénou, 1982). En outre, de telles conditions sembleraient être réunies en galerie forestière et pourraient de ce fait expliquer les fortes densités des diverses espèces de

\section{CONCLUSION}

Cette étude a permis d'identifier dans la région de Ndendé, quatre espèces de glossines, parmi lesquelles G. nashi, G. frezili, et G. palpalis palpalis et $G$. fuscipes fuscipes, vecteurs biologiques de la THA. La répartition de ces espèces de glossines a été corrélée aux biotopes avec une forte abondance dans la galerie forestière comparée à la savane. Aussi, la présence concomitante des animaux domestiques et sauvages, réservoirs potentiels de trypanosomes et des glossines, glossines qui y ont été observées (Foil et Gorham, 2000 ; Solano et al., 2010. Mounioko et al., 2017). Ces résultats corroborent ceux obtenus par Mounioko et al. (2015) qui ont montré une forte abondance des glossines dans les forêts du Parc National de Moukalaba Doudou attenantes à notre zone d'étude. Desquesnes et al. (2005) ont montré que l'importance qualitative et quantitative de l'entomofaune en un lieu donné est définie par ces divers facteurs abiotiques et biotiques. Les résultats de cette étude ont montré que la galerie forestière et la savane ont un fond entomologique commun en ce qui concerne les glossines. En effet, toutes les espèces de glossines capturées dans la galerie forestière ont été aussi retrouvées dans la savane. La similarité entre ces fonds entomologiques serait liée au fait que les deux milieux sont contigus l'un de l'autre et, par conséquent, subissent relativement les mêmes facteurs écologiques. Les espèces de mouches identifiées dans cette zone d'étude ont été essentiellement des glossines anthropophiles, zoophiles ou ubiquistes. Aussi, la présence de G. palpalis palpalis et de $G$. fuscipes fuscipes, vecteurs majeurs de la THA pourrait probablement constituer un risque de transmission de cette parasitose, notamment en zone de forêt. En effet, l'aire de répartition des trypanosomoses est directement liée à celle de son vecteur, la glossine (Brun et al., 2010). Ces résultats sont en accord avec ceux obtenus par Mbida et al. (2009) qui ont montré que les forêts galeries constituent les principaux biotopes propices au développement de $\mathrm{G}$. fuscipes fuscipes où cette espèce trouve des possibilités de repos et de reproduction. La présence des autres espèces comme $G$. nashi, G. frezili, et G. palpalis palpalis traduit leur ubiquité et leur affinité pour les zones de forêt et de savane (Kohagne et al., 2011). Ces résultats sont identiques à ceux obtenus par Mounioko et al. (2015) qui ont révélé la présence de ces quatre espèces dans les forêts et les savanes du Parc National de Moukalaba Doudou, proches de la région de Ndendé.

particulièrement G. fuscipes fuscipes et $G$. palpalis palpalis (vecteurs majeurs des trypanosomes africaines humaines et animales), pourraient entrainer la réémergence des trypanosomoses dans cet ancien foyer de THA. Ainsi, ces résultats invitent à la réalisation des travaux plus détaillés visant à identifier la dynamique spatio-temporelle des glossines et leur rôle dans la transmission éventuelle de la THA dans la région de Ndendé au sud du Gabon. 


\section{REMERCIEMENTS}

Le présent travail a été réalisé grâce à l'appui financier, institutionnel et logistique l'Institut de Recherche en Écologie Tropicale (IRET), du Laboratoire d'Écologie Vectorielle (LEV-IRET), de I'Université des Sciences et Techniques de Masuku, l'École Régionale PostUniversitaire d'Aménagement et de Gestion Intégrée des

\section{BIBLIOGRAPHIE}

Aksoy S., 2003. Control of tsetse flies and trypanosomes using molecular genetics. Veterinary Parasitology, 115 (2) : 125-145.

Allou K., Acapovi-Yao G., Mavoungou J.F., Koumba Z.C.R., 2015. Structuration génétique de trois populations de Glossina palpalis palpalis et impact de la peinture insecticide inesfly ${ }^{\mathrm{TM}} 5 \mathrm{~A}$ IGR NG dans la lutte contre ces vecteurs à Abidjan (Côte d'Ivoire). International Journal of Biological and Chemical Sciences, 9 (2) : 10251037.

Amblard J., 1996. Situation de la trypanosomiase humaine africaine au Gabon. Étude sur la période 1993-1995. Rapport interne du service des grandes endémies, Ministère de la santé, Gabon, 18p.

Amsler S. et Filledier J., 1994. Attractivité pour les Tabanidae de l'association métacrésol/octénol: Résultats obtenus au Burkina Faso. Revue Élevage et de Médecine vétérinaire des Pays Tropicaux., 47 (1) : 93-96.

Bosson-Vanga A.H., Acapovi-Yao G., Kaba D., Dofini F., Coulibaly B., N'dri L., Koné M., 2012. Infection de Glossina palpalis palpalis par les trypanosomes le long du fleuve Comoé dans la région d'Abengourou (Côte d'Ivoire). Journal des Sciences Pharmaceutiques et Biologiques, 13 (1) : 31-37.

Broce A.B., Hogsette J., Paisley S., 2005. Winter feeding sites of hay in round bales as major developmental sites of Stomoxys calcitrans (Diptera : Muscidae) in pastures in spring and summer. Journal of Economic Entomology, 98 (6): 2307-2312.

Brun R, Blum J, Chappuis F, Burri C., 2010. Human African trypanosomiasis. Lancet, 375: 148-59.

Brunhes J., Cuisance D., Geoffroy B., Hervy J.P., 1998. Les glossines ou mouches Tsétsé : Logiciel d'identification et d'enseignement. Paris (FRA) ; Montpellier: ORSTOM ; CIRAD, 1 CD (Didactiques), 7099: 1379-1378.
Forêts et Territoires Tropicaux (ERAIFT) et la CEEAC/PACEBO. Les auteurs remercient vivement Aimé Gildas IBOUILY, Elvy MOUNDOUNGA, Modeste KOUKIDI, Sylvain INGOUDOU et Brice MBADINGA pour leur assistance technique sur le terrain.

Collinet J. et Forget A., 1976. Carte pédologique de reconnaissance. Feuille Booué nord -Mitzic sud. O.R.S.T.O.M. Note explicative, 63, $159 \mathrm{p}$.

Courtin F., Dupont S., Zeze D.G., Jamonneau V., Sané B., Coulibaly B., Cuny G., Solano P., 2005. Trypanosomose Humaine Africaine: Transmission urbaine dans le foyer de Bonon (Côte d'Ivoire).Tropical Medecine and International Health, 10 (4) : 340-346.

Desquesnes M., Dia M.L., Acapovi G.L., Yoni W., Foil L., Pin R., 2005. Les vecteurs mécaniques des trypanosomoses animales : Généralités, morphologie, biologie, impacts et contrôle. Identification des espèces les plus abondantes en Afrique de l'Ouest. Bobo-Dioulasso, Burkina Faso, Éditions Cirdes, $68 \mathrm{p}$.

Dibakou E.S., Mounioko F., Koumba Z.C.R., Nguema M.O.A., Acapovi-Yao G., Mavoungou J. F., 2015. Distribution des Glossines vecteurs de la Trypanosomose humaine africaine dans le Parc National de Moukalaba Doudou (Sud-ouest Gabon). Journal of Applied Biosciences, 86: 7957- 7965.

Djiteye A., 1992. Aperçu sur l'efficacité comparative de différents pièges et odeurs contre les mouches piqueuses (Diptera : Tabanidae et Muscidae) d'importance vétérinaire. In Premier séminaire International sur les Trypanosomoses Animales non Transmises par les Glossines, Annecy (France), 14-16 octobre.

Doumba N.A.G., Zinga K.C.R., Mounioko F., Mbang N.O.A., Mboye B., Maroundou A.P., AcapoviYao G.L., M'batchi B., Mavoungou J.F., 2016. Composition et diversité spécifique des glossines, vecteurs biologiques de la trypanosomose humaine africaine, dans la région de Ndendé (sud du Gabon). Entomologie Faunistique, 69 : 111-123.

Edou E.G., 2012. Diagnostic agro socio-économique de la zone de Lébamba-Ndendé (province de la Ngounié), en vue de la mise en œuvre des 
activités du Prodiag. Institut Gabonais D'appui au Développement. Rapport d'activités. 52p.

Foil L.D. et Gorham J.R., 2000. Mechanical transmission of disease agents by arthropods. In Medical Entomology, Eldridge BF, Edman JD (eds). Kluwer Academic Publishers: Dordrecht, the Netherlands, 461-514.

Gilles J., David J.F., Duvallet G., de La Rocque S., Tillard E., 2007. Efficiency of traps for Stomoxys calcitrans and Stomoxys niger on Reunion Island. Medical and Veterinary Entomology, 21 (21) : 65-69.

Gilles J., 2005. Dynamique et génétique des populations d'insectes vecteurs. Les stomoxes, Stomoxys calcitrans et Stomoxys niger niger dans les élevages bovins réunionnais. Thèse de doctorat, Université de La Réunion, St-Denis, 140p.

Gouteux J.P. et Kiénou J.P., 1982. Observations sur les glossines d'un foyer forestier de trypanosomiase humaine en Côte d'Ivoire. 5. Peuplement de quelques biotopes caractéristiques : Plantations, forêts et galeries forestières, en saison des pluies. Cahiers ORSTOM, Série Entomologie Médicale et Parasitologie, 20 (1) : 41-61.

Jaenson T.G., Dos Santos R.C., Hall D.R., 1991. Attraction of Glossina longipalpalis (Diptera: Glossinidae) in Guinea-Bissau to odorbaited biconical traps. Journal of Medical Entomology, 28 (2) : 284-286.

Kohagne T. L., Gounoue K. R., Mengue M. P., Kaba D., Louis F. J., Mimpfoundi R., 2011. Enquête entomologique dans le foyer historique de trypanosomose Humaine Africaine de Bendjé (Gabon). Parasite, 18 (4) : 303-309.

Laveissière C. et Hervouët J.P., 1991. La trypanosomiase humaine en Afrique de l'ouest. Epidémiologie et contrôle. $2^{2 \mathrm{me}}$ Edition ORSTOM collection Didactiques, Paris, France.

Laveissière, C. et Grébaut P., 1990. Recherche sur les pièges à glossines (Diptera: Glossinidae). Mise au point d'un modèle économique : le piège «Vavoua». Tropical Medicine and Parasitology, 41 (2) : 185-192.

Mavoungou J.F., 2007. Écologie et rôle vecteur des stomoxes (Diptera : Muscidae) au Gabon. Thèse de doctorat. Université Montpellier III Paul Valery. $137 p$.

Mavoungou J.F., Makanga B., Acapovi-Yao G., Desquesnes M., M'batchi B., 2012. Chorologie des Tabanidae (Diptera) dans la réserve de
Biosphère Ipassa-Makokou (Gabon) en saison des pluies. Parasite, 19 (2) : 165-171.

Mbida M.J.A., Mimpfoundi R., Njiokou F., Manga L., Laveissière C., 2009. Distribution et écologie des vecteurs de la trypanosomose humaine africaine de type savanicole en zone de forêt dégradée au sud Cameroun : cas du foyer de Doumé. Bulletin de la Société Pathologique Exotique, 102 (2) : 101-105.

Mihok S., Kangethe E.K., Kamau G.K., 1995. Trials of Traps and Attractants for Stomoxys spp. (Diptera, Muscidae). Journal of Medical Entomology, 32 (3): 283-289.

Milleliri J.M., Kohagne T.L., Mengue M.P., Louis F.J., 2009. La maladie du sommeil au Gabon. Cent cinquante ans d'une histoire contrastée. 18612008. Sciences et Médecine d'Afrique, 2: 119132.

Mounioko F., Dibakou F.S., Zinga-Koumba C. R., MbangNguema O.A., Acapovi-Yao G., Mutambwe S., Mavoungou J.F., 2015. Rythme d'activité journalière de Glossina fuscipes fuscipes, vecteur majeur de la trypanosomiase humaine africaine dans le parc national de Moukalaba Doudou (Sud-Ouest Gabon). International Journal of Biological and Chemical Sciences, 9 (1): 419-429.

Mounioko F., Mavoungou J.F., Zinga K.C.R., Engo P.E., Koumba A.A, Maroundou A.P., Nzengue E., Tamesse J.L., Simo G., M'Batchi B., 2017. Étude préliminaire des vecteurs mécaniques des trypanosomes dans la localité de Campo et ses environs (sud-ouest du Cameroun). Entomologie faunistique-Faunistic Entomology, 70 : 95-105.

OMS-Organisation Mondiale de la Santé, 2015. Trypanosomose humaine africaine (maladie du sommeil). Aide-mémoire N²59, Mai.

OMS-Organisation Mondiale de la Santé, 2016. Maladies à transmission vectorielle. Aide-mémoire №387, Février.

Peka M., Kohagne T.L., Ndeledje N., Louis F.J., Mahamat H.H., 2014. Transmission concomitante de trypanosome humaine et animale : le foyer de Mandoul au Tchad. Revue d'Élevage et de Médecine Vétérinaire des Pays Tropicaux, 67 (1) : 5-12.

Pollock J.N., 1982. Manuel de lutte contre la mouche Tsé-tsé. Volume 1 : Biologie, systématique et répartition des tsé-tsé. FAO, Rome (Italie), 310p.

Raymond H.L., Taufflieb R., Cornet M., Camicas J.L., Chateau R., Dieng P.Y., 1980. Listes annotées 
des Tabanidae (Diptera) du Sénégal et de la Gambie. Bulletin de I'Institut Fondamental d'Afrique Noire, Série A., 42 (4): 812-822.

Seck M.T., Bouyer J., Sall B., Bengaly Z., Vreysen M.J.B., 2010. The prevalence of African animal trypanosomoses and tsetse presence in Western Senegal. Parasite, 17 (3): 257-265.

Shannon C.E., 1948. A mathematical theory of communication. Bell System Technical Journal, 27 (379-423): 623-656.

Sima O.R.L., Zinga K.C.R., Koumba A.A., Nzengue E., Mounioko F., Normark B., Mavoungou J.F., M'Batchi B., 2018. Abondance et distribution des cochenilles sur les feuilles des plantes-hôtes dans la parcelle forestière permanente de Rabi, Sud-Ouest du Gabon. Afrique Science, 14 (1) : 119-128.

Simpson E.H., 1949. Measurement of diversity. Nature, 163: 688-698.

Solano P., Bouyer J., Itard J., Cuisance D., 2010. The cyclical vectors of trypanosomis. Infec and para diseases of livestock, 13: 155-183.

WHO, 2006.Weekly epidemiological record - Relevé Epidémiologique hebdomadaire, 81 (8): 69-80.

Zinga K.R.C., Bouyer J., Mavoungou J.F., Acapovi-Yao G.L., Kohagne T.L., Mbang N.O.A., Ondo K.P.O., Mutambwe S., 2013a. Évaluation de la diversité des diptères hématophages dans une clairière marécageuse du Gabon à l'aide des pièges Vavoua et Nzi. Revue d'Élevage et de Médecine Vétérinaire des Pays Tropicaux, 66 (3) : 91-96.

Zinga K.R.C., Acapovi-Yao G.L., Mavoungou J.F., Tongue K L., Mbang-Nguema O.A., Obame O.K.P., Shango M., 2013b. Influence de la saison sur l'écodistribution des glossines, tabanides, stomoxes du Baï de Momba Makokou, Gabon. Agronomie Africaine., 25 : 149-158.

Zinga-Koumba C.R., Mbang Nguema O.A., Kohagne T.L., Acapovi-Yao G.L., Obame O.K.P., Mutambwe S., Mavoungou J.F., 2014. Contribution à l'évaluation de la diversité des vecteurs biologiques de la Trypanosomose Humaine Africaine et de leur activité journalière dans le Parc National de l'Ivindo (Nord-est Gabon). Journal of Applied Biosciences, 80: 7060-7070.

Zinga K.C.R, Mbang Nguema O.A, Acapovi Yao G.L., Shango M., M'batchi B., Mavoungou J.F., 2015. Étude préliminaire de l'infestation des glossines par les trypanosomes dans le baï de Momba (Nord-est Gabon). Agronomie Africaine, 27 (2) : 95-104. 